of the rational terms of the two-point formulae (10) and (15), on the other hand, are of constant sign throughout the computation.

Acknowledgment. Mr. Shaw's share of the work of this paper was done whilst he was in receipt of a Carnegie Scholarship.

Department of Applied Mathematics

University of St. Andrews

St. Andrews, Scotland

1. P. Brock \& F. J. MUrRaY, "The use of exponential sums in step by step integration," MTAC, v. 6, 1952, pp. 63-78. MR 13, 873.

2. W. GAUTSCHI, "Numerical integration of "ordinary differential equations based on trigonometric polynomials," Numer Math., v. 3, 1961, pp. 381-397. MR 25 *1647.

3. E. REMEs, "Sur le calcul effectif des polynomes d'approximation de Tchebycheff," C. R. Acad. Sci. Paris, v. 199, 1934, pp. 337-340.

4. H. MAEhLY, "Methods for fitting rational approximations," Parts II \& III, J. Assoc. Comput. Mach., v. 10, 1963, pp. 257-277. MR $28 * 707$.

5. J. STOER, "A direct method for Chebyshev approximation by rational functions," J. Assoc. Comput. Mach., v. 11, 1964, pp. 59-69.

6. P. WYNN, "Uber einen Interpolations-Algorithmus und gewisse andere Formeln, die in der Theorie der Interpolation durch rationale Funktionen bestehen," Numer. Math., v. 2, 1960, pp. 151-182. MR $23 *$ B1636.

7. $Z$. KOPAL, "Operational methods in numerical analysis based on rational approximation," Proceedings of a Symposium on Numerical Approximation (1958), R. E. LANGER (ED.), Publication No. 1, Mathematics Research Center, Univ. of Wisconsin, Univ. of Wisconsin Press, Madison, 1959, pp. 25-43.

8. J. D. LAmbert \& A. R. Mitchell, "On the solution of $y^{\prime}=f(x, y)$ by a class of high accuracy difference formulae of low order," $Z$. Angew. Math. Phys., v. 13, 1962, pp. 223-232. MR $25 * 3610$.

\title{
The Numerical Solution of Eigenvalue Problems
}

\section{By Theodore R. Goodman}

1. Introduction. One method for solving eigenvalue problems on a digital computer is to convert the governing differential equations to finite difference equations, apply the boundary conditions at either end of the interval, and form a secular equation for the unknown parameter (the eigenvalue) by setting the determinant associated with the resulting set of homogeneous algebraic equations for the ordinates of the solution equal to zero. Another way of solving eigenvalue problems is to use the Galerkin method. This consists of assuming the solution to be expanded in a complete set of functions satisfying the boundary conditions; upon introducing the series into the differential equation and requiring the error to be orthogonal to the functions in the set there results an infinite set of homogeneous equations for the coefficients. The secular equation is formed by setting the associated determinant equal to zero. These formulations invariably require the determination of the roots of a determinant of large order. The methods arise naturally out of the very nature of an eigenvalue problem and are seen to utilize the capability of digital computers to manipulate matrices of large order.

A completely different method for solving eigenvalue problems will be presented

Received November 23, 1964. This work was supported by the Office of Naval Research under Contract No. Nonr-4432(00). 
here whick expioss the capability of such machines to solve initial value problems. The method is: based on a procedure presented by Goodman and Lance [1] for solving two-poin houdary value problems. In fact, this paper may be considered as a supplement to $[!]$, although it can be read independently.

2. The Eigenvalue Problem. A general system of $n$ linear, ordinary, homogeneous, dificerenial equations may be written

$$
\dot{y}_{i}=a_{i j}(t, \lambda) y_{j}, \quad i=1 \cdots n,
$$

where the dot denotes differentiation with respect to the independent variable $t$, and it is understood that repeated indices are to be summed. The parameter $\lambda$ is the eigenvalue to be determined.

Suppose the boundary conditions to be such that, at $t=0, r$ of the $y_{i}$ 's, viz.: $y_{1}(0), \cdots, y_{r}(0)$ are zero, and at $t=T,(n-r)$ of the $y_{i}$ 's, viz.: $y_{i_{k}}(T)$, $k=1 \cdots(n-r)$, are zero.

These homgeneous differential equations and boundary conditions constitute the cigenvalue problem.

3. The Method of Solution. For a linear eigenvalue problem, the eigenfunctions arf unknown to within a muitiplicative constant. Hence, it is permissible to choose

$$
y_{r+1}(0)=1,
$$

which fixes this constant. Of course, if the eigenfunctions must be normalized in a certain way an adjustment may be made at the end of the calculation. The procedure is to estimate the remainder of the initial conditions $y_{r+2}(0), \cdots, y_{n}(0)$ as well as the eigenvalue $\lambda$; these estimates are denoted by $y_{r+2}^{*}(0), \cdots, y_{n}{ }^{*}(0), \lambda^{*}$. Equations (1) can now be integrated as an initial value problem, and the solutions denoted by $y_{i}{ }^{*}(t)$. In general, it will be found that the computed values of $y_{i_{k}}(T)$, $k=1, \cdots,(n-r)$, namely, $y_{i_{k}}^{*}(T)$, differ from zero. To obtain the correct solution the values of $y_{i_{k}}^{*}(T)$ must be made as small as possible.

Define $\delta y_{i}(t)$ by

$$
\delta y_{i}(t)=y_{i}(t)-y_{i}^{*}(t), \quad i=1 \cdots n,
$$

and, at the same time,

$$
\delta \lambda=\lambda-\lambda^{*} .
$$

Substituting (3) and (4) into (1), there results to a first approximation

$$
\delta \dot{y}_{i}=a_{i j}\left(t, \lambda^{*}\right) \delta y_{j}+\frac{\partial a_{i j}}{\partial \lambda}\left(t, \lambda^{*}\right) \delta \lambda y_{j}^{*}
$$

These are the equations of differential corrections. The equations adjoint to them are

$$
-\dot{x}_{i}=a_{j i}\left(t, \lambda^{*}\right) x_{j}
$$

where the $\delta y_{i}^{\prime}$ 's and the $x_{i}$ 's are related by

$$
x_{i}(T) \delta y_{i}(T)-x_{i}(0) \delta y_{i}(0)=\delta \lambda \int_{0}^{T} \frac{\partial a_{i j}}{\partial \lambda}\left(t, \lambda^{*}\right) y_{j}^{*} x_{i} d t .
$$


The quantities required are $\delta y_{r+2}(0), \cdots, \delta y_{n}(0), \delta \lambda$, from which improved estimates of $y_{r+2}(0), \cdots, y_{n}(0), \lambda$ can be obtained. To obtain $(n-r)$ equations for these $(n-r)$ unknowns, $(6)$ is integrated from $T$ to $0(n-r)$ times; the result of the $m$ th integration is denoted by ${ }_{m} x_{i}(t)$. Equation (7) holds for each integration of the adjoint system, and this may be expressed by rewriting it as

$$
{ }_{m} x_{i}(T) \delta y_{i}(T)-{ }_{m} x_{i}(0) \delta y_{i}(0)=\delta \lambda \int_{0}^{T} \frac{\partial a_{i j}}{\partial \lambda}\left(t, \lambda^{*}\right) y_{j}{ }^{*} x_{i} d t .
$$

The $m$ th time the adjoint equations are integrated, the starting values are chosen so that all the $x_{i}(T)$ 's are zero except the coefficient of $\delta y_{i_{m}}$, which is taken to be unity. The first summation in (8) then reduces to a single term. Furthermore, in order for the homogeneous conditions at $t=T$ to be satisfied, $\delta y_{i_{m}}(T)$ must be set equal to $-y_{i_{m}}^{*}(T)$. In addition, since the initial data for $y_{i}, i=1 \cdots r+1$, remain unperturbed, $\delta y_{i}(0), i=1 \cdots r+1$, are identically zero. Thus (8) becomes

$$
-y_{i_{m}}^{*}(T)-{ }_{m} x_{k}(0) \delta y_{k}(0)=\delta \lambda \int_{0}^{T} \frac{\partial a_{i j}}{\partial \lambda}\left(t, \lambda^{*}\right) y_{j}^{*}{ }_{m} x_{i} d t
$$

where the summation on $k$ extends from $r+2$ to $n$. Substituting the value of $\delta \lambda$ into (4), and the values of $\delta y_{k}(0)$ into (3), improved values of $y_{i}(0)$ and $\lambda$ are obtained. The procedure is then repeated until convergence is achieved. The final value of $\lambda$ is the eigenvalue, and the final functions $y_{i}(t)$ are the eigenfunctions (which may have to be normalized).

It should be pointed out that the method of differential corrections is equivalent (though more elaborate) to Newton's method for obtaining the roots of a transcendental equation, and, hence, there is no guarantee that convergence will occur. Futhermore, unless the initial estimates for the $y_{i}{ }^{*}(0)$ 's and $\lambda^{*}$ are in the correct neighborhood it is possible to converge to some other eigenvalue (if such exists) than the one sought.

4. An Example. Consider the second-order differential equation

$$
\frac{d^{2} y}{d t^{2}}+\lambda^{2} y=0
$$

subject to the boundary conditions $y(0)=y(1)=0$. The eigenvalues are known to be $\lambda=n \pi, n=1,2,3 \cdots$. In order to apply the method of this paper, the equation is first written in tensor form:

$$
\begin{aligned}
& \dot{y}_{1}=y_{2}, \\
& \dot{y}_{2}=-\lambda^{2} y_{1}
\end{aligned}
$$

subject to the boundary conditions $y_{1}(0)=y_{1}(1)=0$. From (2) we assume initial data $y_{1}(0)=0, y_{2}(0)=1$. Since the equation is only of second order it is not necessary to estimate any initial data whatsoever; the only unknown parameter is $\lambda$ itself. The perturbation equations are

$$
\begin{aligned}
& \delta \dot{y}_{1}=\delta y_{2}, \\
& \delta \dot{y}_{2}=-\lambda^{* 2} \delta y_{1}-2 \lambda^{*} \delta \lambda y_{1}{ }^{*} .
\end{aligned}
$$


The equations adjoint to these are

$$
\begin{aligned}
& -\dot{x}_{1}=-\lambda^{* 2} x_{2}, \\
& -\dot{x}_{2}=x_{1},
\end{aligned}
$$

which are to be solved only once subject to the initial conditions $x_{1}(1)=1$, $x_{2}(1)=0$.

For this problem, $(9)$ is only one equation, viz.,

$$
-y_{1}^{*}(1)=-2 \lambda^{*} \delta \lambda \int_{0}^{1} y_{1}^{*} x_{2} d t
$$

The solutions for this simple case can easily be generated analytically. The solution of (11) subject to the given initial data is

$$
y_{1}^{*}=\frac{\sin \lambda^{*} t}{\lambda^{*}}, \quad y_{2}^{*}=\cos \lambda^{*} t
$$

The solution of (12) subject to its initial data is

$$
x_{1}=\cos \lambda^{*}(1-t), \quad x_{2}=\frac{\sin \lambda^{*}(1-t)}{\lambda^{*}} .
$$

Substituting (15) and (16) into (14) and solving for $\delta \lambda$ yields

$$
\delta \lambda=\frac{\lambda^{*} \sin \lambda^{*}}{\sin \lambda^{*}-\lambda^{*} \cos \lambda^{*}} .
$$

'That the method is truly equivalent to Newton's method for finding roots is demonstrated by this equation, for, by applying Newton's method to the function $(\sin \lambda) / \lambda$, a differential correction identical to (17) is obtained. To give an idea of the rapidity of convergence, (17) was applied starting with the estimated value $\lambda^{*}=1$. The results of successive iterations are shown in Table I.

5. Remarks. The problem was posed such that the $y_{i}$ 's were given at either end of the interval $0-T$. The method is easily extended if, instead, $n-p$ homogeneous linear relations are prescribed between the $y_{i}(0)$ 's and $p$ between the $y_{i}(T)$ 's. Indeed, these relations may even involve the unknown eigenvalue $\lambda$.

The possibility of solving initial value problems for the purpose of solving eigenvalue problems was first presented by Fox [2]. The basic difference between his method and the one presented here is that Fox works directly with the equations of differential correction which are nonhomogeneous, whereas, in the present

TABLE I

Results of Successive Iterations of (17)

1.00000

3.79400

2.83711

3.12001

3.14145

3.14159 
method, the adjoint equations are homogeneous and the nonhomogeneity is postponed to the algorithm (9) for obtaining the corrections. As to which method is faster or more efficient, it is not possible at this time to say. Some time later Brown [3], in connection with a problem in the theory of hydrodynamic stability, independently introduced a method using initial value problems, but convergence to the solution was achieved partially by trial and error so that the method is not fully automatic. This objection was overcome by Nachtsheim [4] who used a perturbation scheme and iterated to the final solution; he was compelled, however, to estimate more constants than are truly required. Although all of these investigators worked on the same problem, none of them seems to have been aware of his predecessors.

The principles of the method presented here may be applied to solve nonlinear eigenvalue problems, since, in solving initial value problems, the computer is indifferent to linearity. Of course, the equations of differential corrections are linear in any case, and so are their adjoints; but in this case the coefficients depend on the previous iteration of the eigenfunctions. In nonlinear cases the solution cannot be arbitrarily normalized because, in contrast to the linear case, the eigenvalues depend on the amplitude of the eigenfunctions (e.g., in determining the period of a cubic spring). In fact, for nonlinear cases, the eigenvalue problem would have to be solved many times in order to grasp this dependence, and the relation between the initial value of $y_{r+1}$ and the amplitude would have to be established from the eigenfunctions. It might be pointed out that nonlinear eigenvalue problems cannot be solved using methods involving a secular equation (except for periodic solutions when one frequency dominates, in which case the method of equivalent linearization can be used as an approximation), and, in this respect, the present method is superior.

Oceanics, Inc.

Plainview, L. I., New York

1. T. R. Goodman \& G. N. LAnce, "The numerical integration of two-point boundary value problems," $M T A C$, v. 10, 1956, pp. 82-86. MR 18, 420.

2. L. Fox, The Numerical Solution of Two-Point Boundary Value Problems in Ordinary Differential Equations, Oxford Univ. Press, New York, 1957; p. 245. MR 21 * 972.

3. W. B. Brown, "Exact solution of the stability equations for laminar boundary layers in compressible flow," Boundary Layer and Flow Control: Its Principles and A pplication, Vol. 2, G. V. Lachmann, Ed., Pergamon Press, New York, 1961; pp. 1023-1038. MR 28 * 1843.

4. P. R. Nachtsheim, An Initial Value Method for the Numerical Treatment of the OrrSommerfeld Equation for the Case of Plane Poiseuille Flow, NASA TN D-2414, August, 1964.

\title{
A Special Technique For The Determination of Eigenvalues
}

\author{
By V. O. S. Olunloyo
}

1. Introduction. We consider the problem of determining an eigenvalue of pre-scribed order of the system

$$
y^{\prime \prime}+[\sigma(x)+\lambda] y=0, \quad y(0)=y(1)=0, \quad \sigma>0 .
$$

We specifically wish to avoid the eigenvalues of lower order. We may begin with a reasoned guess based partly on classical inequalities. The problem then boils down

Received July 6, 1964. Revised November 10, 1964. 